\title{
DEL HOGAR A LA CIUDAD COMO CAMINO DE IDA Y VUELTA EN EL DESARROLLO DE LA IDENTIDAD: EL CASO DE LAS RUTINAS DE LA INFANCIA URBANA DE CLASE MEDIA/ALTA EN MADRID
}

\author{
Marta Morgade Salgado* \\ David Poveda Bicknell* \\ Javier González-Patiño*
}

\begin{abstract}
RESUMO: Se viene definiendo el hogar como un espacio dinámico (POVEDA et al., 2012), que no forma parte únicamente de lo público o lo privado, los límites entre ambos se vuelven más difusos, ya que los medios digitales ponen al alcance el espacio público dentro del entorno privado. Presentamos un estudio cualitativo de las rutinas y el uso que la infancia hace de los recursos que su entorno les proporciona. Se realizó con 4 menores de entre 9-13 ańos de 2 distritos de clase alta de Madrid. El análisis muestra cómo reparten su tiempo entre el espacio público y privado, cómo hacen uso de los recursos a tienen acceso y que les proporciona el hogar, y a su vez la familia, ambos ofrecen un amplio acceso al capital económico y cultural (BOURDIEU, 1994), asociado a su clase socioeconómica. (LAREAU, 2002)
\end{abstract}

Palabras clave: Rutinas Infantiles. Ciudad. Capital Cultural. Familia. Medios Digitales.

\section{FROM HOME TO THE CITY AS A WAY OF COMING AND GOING IN THE DEVELOPMENT OF IDENTITY: THE CASE OF MEDIUM/HIGH CLASS URBAN CHILDHOOD ROUTINES IN MADRID}

ABSTRACT: Home has been defined as a dynamic space (POVEDA et al, 2012), which is not just part of either the public or the private space. The boundaries between these spaces become more diffuse, since digital media place the public space within the private environment. We present a qualitative study of children's routines and the use of resources that their environment provides. It was conducted with 4 children aged between 9-13, in 2 upper-class districts of Madrid. The analysis shows how they share their time between public and private spaces, how they make use of the resources they have access to, and what

\footnotetext{
* Universidad Autónoma de Madrid, Facultad de Formación de Profesorado y Educación, Departamento Interfacultativo de Psicología Evolutiva de la Educación, Madrid, España. E-mail de contacto: mmorgade.s@gmail.com.
} 
their homes, and in turn their families, provide to them; both offer broad access to economic and cultural capital (BOURDIEU, 1994) associated with their socioeconomic class. (LAREAU, 2002)

Keywords: Children`s routines. City. Cultural Capital. Family. Digital media.

\section{DE LA MAISON À LA VILLE COMME UN MOYEN D`ALLER ET VOLER DANS LE DÉVELOPPEMENT DE L'IDENTITÉ: LE CAS DES ROUTINES DE L`ENFANCE URBAINE CLASSE MOYENNE/ HAUTE DE MADRID}

RÉSUMÉ: La maison a été définie comme un espace dynamique (POVEDA et $a l, 2012$ ), qui n'est pas exclusivement un espace public ni privé, les frontières entre les deux deviennent plus diffuse, car les médias numériques mettent accessible l'espace public dans l'environnement privé. Nous présentons une étude qualitative des routines et de l'usage des enfants qui s'en utilise des ressources offertes par l'environnement. L'étude a été réalisée avec 4 enfants âgés de 9 à 13 ans, de 2 districts de la classe privilégiée de Madrid. L'analyse montre comment ils partagent leur temps entre l'espace public et privé, comment ils font l'usage des ressources pour en avoir l'accès et les fournir à la maison, et à son tour, à la famille, les deux offrent un large accès au capital économique et culturel (BOURDIEU, 1994) associés à la classe socio-économique. (LAREAU, 2002)

Mots-clés: Routines des enfants. Ville. Capitale culturelle. Famille. Médias numériques.

\section{Introducción}

ás de la mitad de los niños en países industrializados habitan en ciudades mientras que el número crece a pasos forzados en países en vías de desarrollo y subdesarrollados. El crecimiento trepidante de las ciudades en ocasiones impide diseñarlas teniendo en cuenta las necesidades de todos los grupos que las habitan; los más desfavorecidos pierden sus derechos a favor de las comodidades de los grupos más fuertes. Quizás el grupo con menos voz en de los proyectos que se realizan en las ciudades sea la infancia. (POVEDA et al, 2007) Pero los espacios públicos se convierten en el lugar en el que aprenden a establecer contacto con otros niños de su edad, sin necesidad de intervención de mayores. Se establecen relaciones con grupos de otras edades de los que se aprende a interactuar, a relacionarse, se aprenden juegos y roles sociales que surgen en el entorno de la calle. (ROGOFF, 2003)

Atendiendo al modelo del triángulo institucional de Rasmussen y Smidt (2003), el tiempo de la infancia se reparte entre el hogar, la escuela y las 
instalaciones recreativas extraescolares. En cambio los espacios públicos no regulados aparecen simplemente como espacios utilizados para la movilidad y los más pequeños los utilizan para trasladarse de un lugar a otro, pero no como espacio en el que pasar el tiempo libre del que disponen. Poveda et al. (2007) mostraron que aunque existe una orientación general similar, hay variedad de recursos, ideas y valores para organizar tiempo y rutinas extraescolares.

Sin embargo casi todos los estudios sobre la infancia y sus procesos de socialización, las prácticas socioeducativas se estudian con relación a las diversas estrategias e ideologías parentales tienen así como las prácticas familiares (POVEDA et al, 2012) y queda fuera de la descripción y comprensión del proceso la forma y el sentido que esas prácticas tienen para los menores.

Se ha prestado atención a las estrategias familiares desde que Coleman (1966) demostró para EEUU que la variable que mejor explicaba los resultados académicos estaba en la tipología de familias, su contexto socioeconómico y las 'oportunidades' educativas que ofrecen, se ha generado un discurso que postula que hay familias "que funcionan" y otras que no. Esto, a su vez, ha dado lugar a la tendencia a pedagogizar el ambiente familiar (DE CARVALHO, 2001) a partir de un modelo de familia de clase media que, proyectado desde las escuelas y ciertas políticas públicas, se usa de molde para cambiar a las familias que son consideradas 'de riesgo'. En este tipo de políticas públicas se afirma que para lograr una mejora del sistema educativo se necesita 'más escuela' en los hogares, y fuera del horario escolar. Pero estos programas no sólo han tenido un alcance limitado, sino que se han documentado efectos no deseados como facilitar más aún la participación de los más privilegiados.

Esta aparente contradicción entre la importancia del factor familia y las limitaciones de las políticas 'participativas' emana de la falta de análisis de las estrategias familiares que están detrás de la reproducción de las desigualdades sociales, incidiendo en los procesos sociales más amplios de legitimación y construcción de los grupos sociales favorecidos. Entorno a las diferentes estrategias familiares, estudios clásicos como los de Bourdieu (1994) o recientes (LAREAU, 2002) hablan la idea de involucramiento parental como patrones regulares de acción, objetivamente orientadas, que pueden o no estar orientadas hacia fines subjetivamente buscados. Se trata de la puesta en práctica de un sentido de percibir, valorar, preferir, ser y hacer, condicionado estructuralmente por el lugar socio-económico que las familias ocupan en la estructura social. Ello genera prácticas, habitus, en las que participan y se socializan las infancias de esas familias, reproduciendo las desigualdades de partida. Lareau (2002) plantea que las familias de clase media norteamericana orientan su acción hacia lo que denomina cultivación concertada concerted cultivation, se caracteriza: fuerte uso del lenguaje, estimulación del razonamiento infantil, la participación de las niñas en actividades extra-escolares variadas, entre otros. Lógicas similares se han descrito también en familias in- 
glesas y francesas de clase media. (VINCENT et al, 2012) Menos conocimiento se ha desarrollado para el caso de las familias españolas. En un estudio reciente (GUBBINS, 2011) se estudiaban estas estrategias familiares en el contexto de la clase media y alta chilena, encontrando matices diferentes entre las formas de las estrategias de involucramiento familiar. Mientras las familias de clase media se posicionan en alianza con las exigencias y reglas del establecimiento educacional, las de clase alta lo hacen desde una lógica de administración desde fuera del establecimiento educacional.

Los estudios mencionados reivindican las estrategias familiares a la hora de gestionar las rutinas infantiles en orden a reproducir ciertos estatus, o en algunos casos a la mejora de ese estatus en el futuro de sus hijos. Todo ello comprende las diversas formas de dar sentido, en las familias, del papel que las prácticas socioeducativas tendrán en el desarrollo de sus hijos. Esas prácticas involucran tanto la escuela, la ciudad, el hogar y otros espacios de manera sistémica. Sin embargo, son estudios que no atienden a las formas de identificación y gestión autónoma de sus prácticas de los menores, puesto que no se acercan a esas prácticas desde el punto de vista de quienes deben reproducir, o no, en sus vidas esos valores.

Si bien la investigación cuantitativa permite establecer indicadores generales de bienestar infantil y obtener información sobre aspectos relacionados con sus tareas diarias, con la metodología cualitativa se intenta, además, entender cómo se enfrentan las nińas a sus entornos, cómo se adaptan e integran los recursos que se les ofrecen y cómo adaptan sus forma de vida en función de aquello a lo que acceden.

Nuestro objetivo en este artículo es obtener una amplia información sobre las rutinas de los infantes dejando que participen de forma activa en la investigación acercándonos así no sólo a las prácticas familiares, los espacios, actividades, personas, también y de manera especial al sentido que esas prácticas tienen para ellas. ¿Cómo son interpretadas, asumidas o discutidas por unos niños que parecen "tener" la tarea de reproducir el orden socioeconómico familiar a partir de sus rutinas diarias? ¿Cómo reconocen, o no, el valor del capital cultural y económico familiar en el que se desarrollan? Recurrimos para ello al uso de materiales auto producidos (fotografías) con consignas generales. Así los niños documentan su día a día, nos permiten entrar en esas actividades sin organizar su narración sin partir de nuestras preguntas, y son los niños quienes desde el material que nos entregan, elicitan nuestras preguntas. Es decir, una metodología así utilizada en otros estudios relacionados con rutinas infantiles (COOK y HESS, 2007; POVEDA, MORGADE y GONZÁLEZ-PATIŃO, 2012) que permite que los niños realicen fotografías con su propia lógica, descubrir sus rutinas desde el su punto de vista en su vida diaria, y participar de activamente en el proceso de investigación. 


\section{Metodología}

Esta investigación usa una muestra de conveniencia compuesta por 4 casos de nińos y pre-adolescentes cercanos a la red social de una de las investigadoras. Todos ellos participaron voluntariamente en el trabajo con consentimiento de sus padres. Los casos de este estudio fueron seleccionados utilizando como criterio principal su disposición a participar en el estudio y por el hecho de que se auto-identificaron como usuarios de diferentes medios digitales, y tenían fácil acceso a ellos en sus hogares. Los 4 casos viven con sus familias en los distritos de Salamanca o Chamartín de Madrid, las 2 áreas de la ciudad con la rentas per cápita más altas. Todos pertenecían a familias de clase alta encabezadas por padres profesionales liberales y en puestos ejecutivos.

La recogida de datos se basa en la entrevista foto-elicitada con la realización de series de fotografías por parte de los participantes. (CAPELLO, 2005; RASSMUSEN, 2004) Los 4 casos se implicaron en 2 series fotográficas y subsecuentes sesiones de entrevista a partir de las fotografías. En la primera serie las consigna iba dirigida a documentar las rutinas generales post/extra-escolares de los 4 participantes y se ofreció la siguiente indicación: "Estoy investigando las rutinas de los niños/as de Madrid, por lo que me interesa que hagas todos los días, durante una semana, al menos 5 fotos diarias sobre las actividades, lugares o situaciones que consideres más importantes para ti”. La segunda serie de fotografías se centró en el uso y relación con la tecnología. Todos los participantes se implicaron activamente y utilizaron sus cámaras fotográficas digitales para generar el corpus de fotografías.

\section{Tabela 1}

Participantes en el estudio (seudónimos)

\begin{tabular}{|c|c|c|c|}
\hline Participante & Edad (al inicio) & Hogar & Tipo escolarización \\
\hline Carola & 13 & $\begin{array}{c}\text { Distrito Salamanca. Carola, } \\
\text { Lucía y madre }\end{array}$ & Centro concertado \\
\hline Lucía & 9 & $\begin{array}{c}\text { Distrito Chamartín. Padres } \\
\text { y 2 hermanos (níno y niña) } \\
\text { Alicia }\end{array}$ & Centro privado que ella \\
Bruno & 12 & $\begin{array}{c}\text { Distrito Chamartín. Padres } \\
\text { y 2 hermanos mayores que } \\
\text { él }\end{array}$ & Centro privado \\
\hline
\end{tabular}


Tras cada serie fotográfica se realizó una entrevista semiestructurada con cada uno de los participantes utilizando las fotografías como eje. La primera entrevista se centraba en explorar los espacios, actividades y sistemas de relaciones sociales que aparecen en la vida cotidiana de los niños fuera de la escuela, así como el significado que tienen estos elementos en su vida. Igualmente, esta entrevista iba encaminada a construir, a través del discurso y los elementos visuales, su narrativa general sobre la rutina semanal global de cada participante. (POVEDA et al, 2007) La segunda entrevista, de formato y estructura similar, se centró en explorar los usos de las tecnologías y el sentido que le atribuyen en su vida diaria los participantes. Todas las entrevistas se realizaron en su hogar u otro espacio de la familia extensa, por lo que como parte de la recogida de los datos se realizaron varias "visitas" (y observaciones) al hogar de los participantes y su entorno inmediato.

Finalmente, la recogida de datos se cerró con una entrevista a los padres en las que se exploraron sus ideologías socio-educativas y el proceso de toma de decisiones en torno a aspectos como la elección de hogar, centro educativo y organización de la vida extra-escolar del hijo/a bajo estudio - aunque en este trabajo, como hemos señalado en la introducción, vamos a presentar y discutir principalmente el punto de vista de los menores. Todos los datos se recogieron entre 2009 y 2010 con varios meses de dilación entre cada sesión de entrevistas fotográficas con los menores y con los padres; de tal manera que el conjunto de datos y observaciones más informales generadas a lo largo del proceso permiten valorar la estabilidad de los datos recogidos en cada fase del proyecto.

\section{Resultados}

Organizaremos los análisis de las formas de vida de los casos bajo estudio en torno a 3 ejes temáticos: (a) la gestión y organización de las rutinas y tareas diarias, (b) la organización de los tiempos y actividades de ocio y (c) las relaciones familiares y con la familia extensa. Estos ejes servirán para dar cuenta del modo en que se moviliza el capital social y simbólico de las familias participantes y las interrelaciones hogar-entorno urbano que se generan con esta organización de la vida diaria. El análisis del papel de la tecnología aparecerá de manera transversal como parte de estos ejes temáticos ya que su lugar específico ya ha sido analizado en otros trabajos. (GONZÁLEZ-PATIÑO, 2011; POVEDA et al, 2012)

A pesar de que los participantes viven en 3 familias diferentes que residen en 2 distritos distintos de la ciudad (aunque cercanos) se dan convergencias en la organización de su vida diaria después de la jornada escolar y los fines de semana que sirven para retratar la organización del entorno en el que están creciendo y los recursos tanto simbólicos como materiales que se movilizan en su vida cotidiana. Este primer análisis de las rutinas diarias pone énfasis en el sistema de "obligacio- 
nes" que queda retratado en las fotografías y entrevistas; es decir, señalando más bien las responsabilidades y compromisos que conforman la vida diaria de las niñas participantes.

\section{Tareas del hogar}

A pesar de su posición socio-económica privilegiada, los 4 participantes dan importancia a aquellos aspectos que tienen que ver con la ayuda en tareas del hogar: quitar la mesa y ponerla, cocinar en algunos casos o hacer la cama. Como hemos seńalado, entienden que estos aspectos entran dentro de sus obligaciones como integrantes de la familia y, además, asumen que tienen que realizar estas tareas de manera autónoma. Igualmente, en su discurso, aunque en general estas actividades surgen de órdenes adultas, los participantes entienden que forman parte de la lógica de las actividades dentro de la casa, que implica también a los demás miembros y dentro de las cuáles cada uno asume un rol determinado. Así lo explica Carola:

Pues que todos los días tengo que poner la mesa con mi hermana $[\ldots]$ ponemos la mesa mientras mi madre cocina. Nos repartimos las tareas entre las $2[\ldots]$ hay veces que si ella tiene más deberes y yo ya he terminado pues la pongo yo, pero luego siempre la quitamos juntas (Carola).

\section{Figura 1}

Fotografía de la ducha de Bruno

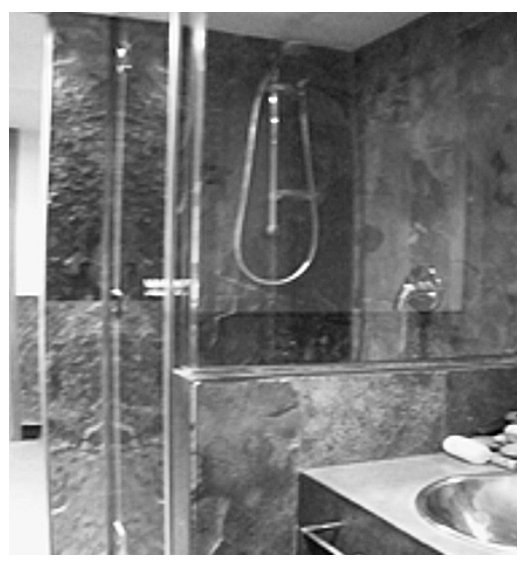


Otras rutinas diarias giran en torno al cuidado personal, la ducha, lavarse los dientes. Tanto Alicia como Bruno mencionan su preocupación por la higiene personal, sentirse limpio y estar arreglado. Factores externos como el aparato dental en el caso de Alicia hacen que tomen conciencia de las rutinas de higiene de manera muy subjetiva. Así, Alicia comenta en la entrevista: "Llevo brackets y a mí los dientes es una cosa que me tengo que cuidar mucho." En la misma línea, Bruno realiza varias fotografías que tienen que ver con la ducha y comenta que para él ducharse es la mejor manera de comenzar el día.

Esta foto la hice por la mañana, porque por las mañanas siempre me ducho para ir al cole limpio y [...] no estar en la cama, estar ahí durmiendo y todo [...] (Bruno).

\section{Actividades extra-escolares "organizadas" y estudio}

Carola y Lucía asisten a un "centro concertado" (gestionado por una entidad privada pero financiado por la administración pública) en el que pasan gran parte de la jornada diaria, entre $8 \mathrm{~h} 30 \mathrm{~min}$. $-17 \mathrm{~h}$. Por su parte, Alicia y Bruno van al mismo colegio privado con un horario continuo de mańana, entre 8h15min.-14h20min., que luego tiene múltiples actividades extra-escolares para sus alumnos. Así, entre semana, Alicia y Bruno comen en su propia casa, en casa de amigos o familiares y tienen la tarde disponible para hacer los deberes y dedicar una parte del tiempo a aquellas actividades estructuradas en las que deciden implicarse o han sido incorporadas a su formación por sus padres. Como ilustración de esta agenda intensamente planificada tenemos el resumen que realiza Bruno de su propio horario:

De lunes a viernes tengo clase de $8 \mathrm{~h} 15 \mathrm{~min}$. a $2 \mathrm{~h} 20 \mathrm{~min}$. Los lunes voy a comer a casa de un amigo y tenemos clases de alemán de $3 \mathrm{~h}$ a $5 \mathrm{~h}$ y luego me vuelvo a casa y hago deberes. Los martes como en el colegio y tengo clase de violín de $3 \mathrm{~h}$ a $4 \mathrm{~h}$ y luego sobre las 5 h o así me vengo a casa. Luego estudio o hago los deberes hasta las 7 h más o menos [...]. Los miércoles voy a casa a comer y luego juego al voleibol en el colegio. Los jueves como en casa y luego tengo clase aquí de pádel. Nos lleva mi madre a mi prima y a mí (Bruno).

Para los 4 casos del estudio los deberes, el estudio de los contenidos académicos después de la jornada escolar y la preparación de exámenes ocupan el lugar más importante y vertebran el tiempo que tienen disponible para otras actividades y su ocio. 
Carola: Los miércoles y los viernes termino a la una, pero los miércoles me quedo en estudio dirigido [...] que nos ponen en mesas de exámenes y hacemos deberes tutelados.

E: ¿Entonces los miércoles cuando llegas a casa no tienes deberes? Carola: pero estudio...o sea a lo mejor sí que me quedan... porque aprovechan para mandar más.

E: ahh

Carola: Aquí hay muchos libros [...] son las asignaturas de las que tengo deberes. Hay gente que se pone horarios pero yo no hago eso porque solo pienso en el descanso. Los ordeno por como tenga las horas al día siguiente. Los hago hasta que se acabe el montón y después estudio.

\section{Figura 2}

Fotografía de los libros escolares de Carola

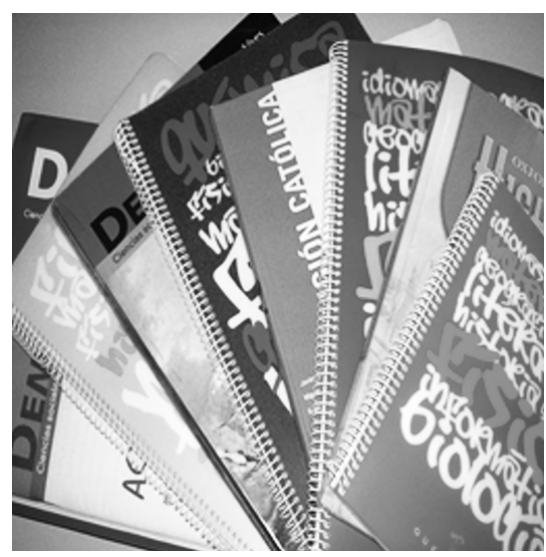

A pesar de que el estudio es visto como una obligación y no necesariamente una actividad placentera, los propios participantes ya empiezan a incorporar en su discurso la importancia de la formación académica en sus futuras trayectorias socio-laborales y visibilizar el papel que se otorga a la formación escolar y académica en la familia. Así, Bruno hace explícita tanto su propia visión del estudio y la postura promovida por sus padres:

[...] estudiar no le gusta a nadie, ¿no? Pero todos saben que es necesario para poder sacar buenas notas y tener un futuro [...] cada uno tiene una mesa de estudio para poder estudiar [...] si, eso sí que mis padres lo ven como muy importante, que no nos 
peleemos por una mesa de estudio porque estudiar es muy importante para que saquemos buenas notas y todo [...] entonces, ellos ven muy importante que tengamos todo lo necesario ¿̨no?, para poder estudiar.

De esta manera, en la habitación de los participantes la "mesa de estudio" cobra una relevancia especial y se convierte en un micro-espacio en el que ejercen mayor control e incluso despliegan sus subjetividades incorporando objetos personales (e. g. libros, música, postales) más allá de los relacionados con el estudio y la vida escolar:

[...] es un sitio donde yo tengo mis cosas, tengo una, mi mesa de estudio, luego, mi mesa de estudio, la ventana donde me entra toda la luz ahí es donde estudio, hago los deberes donde me da todo igual, es mi sitio donde entran por alguna cosa en especial, hombre tengo ahí los libros y si quieren tal, pero es un sitio muy especial [...] mi iPod, mi móvil, todo lo tengo allí (Alicia).

Igualmente, en este espacio de estudio y las "rutinas de deberes", los menores hacen uso de diferentes dispositivos, no sólo los libros de texto. El pc e Internet para hacer trabajos o para consultas y el móvil para comunicarse con los compañeros de la escuela aparecen de manera reiterada. (GONZÁLEZ-PATIÑO, 2011) Los dispositivos utilizados pueden pertenecer tanto al propio nińo/a, como en el caso de Alicia; ser de uso compartido, como hace Bruno con sus hermanos o de los padres y como es el caso de Carola y Lucía. En todos los casos, independientemente de la distribución de los dispositivos, su uso para actividades relacionadas con la escuela está permitido y no es cuestionado por los padres, que sin embargo, como veremos más adelante son más "intrusivos” en otros aspectos relacionados con el uso de las nuevas tecnologías.

Los deberes ocupan la mayor parte del tiempo de las actividades de la tarde durante la semana laboral: los 4 participantes mencionan que tan sólo se pueden hacer otras actividades de ocio como ver la televisión o conectarse al ordenador en caso de que aquellas que tienen que ver con la escuela estén terminadas. Además, aunque cabe destacar que todos reciben clases de refuerzo relacionadas con asignaturas escolares en el hogar. En el caso de Bruno y Alicia, las clases son de refuerzo de alemán y están condicionadas por el centro educativo al que asisten; mientras que Carola y Lucía asisten a clases particulares de matemáticas.

Finalmente, más allá del tiempo dedicado al estudio y actividades directamente relacionadas con el "refuerzo" de las materias curriculares, participan en actividades extra-escolares que amplían sus aprendizajes y experiencia formativa. Estas actividades comienzan dentro de las instalaciones escolares y pueden exten- 
derse al espacio doméstico y otros tiempos de ocio - solapándose o creando, como veremos más abajo, espacios de ocio-tiempo libre estructurados. Así, Bruno, toca el violín en un grupo de la escuela y practica después en casa, mientras que Lucía juega al ping-pong y al bádminton en las instalaciones escolares los viernes por la tarde.

\section{Figura 3}

Bruno practicando violín en casa

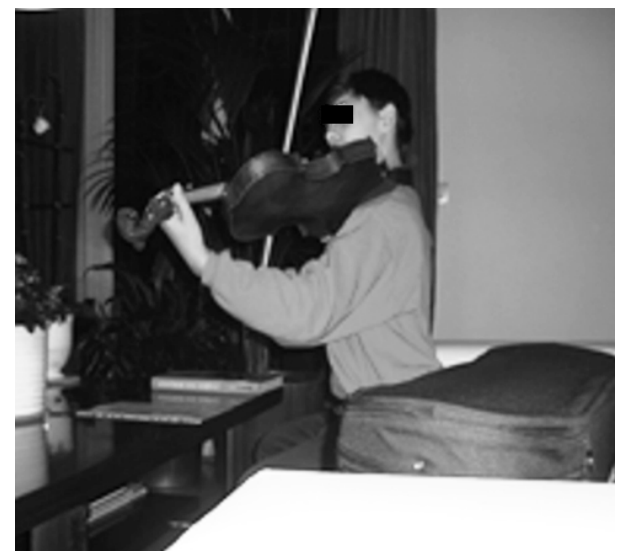

Los casos analizados también disponen de "tiempo libre" con actividades que eligen libremente. Estas actividades tienen diferentes grados de estructuración y vinculación con otras instituciones, organizaciones e incluso en centro educativo.

\section{La práctica de deporte}

Después de los deberes y las tareas relacionadas con la escuela ya descritas surge el deporte como una de las actividades más relevantes a lo largo de la semana. Éste puede practicarse en instalaciones recreativas cercanas a la casa, en el caso de Carola y Lucía, o en clubs deportivos en el caso de Alicia y Bruno. En este sentido, los 4 participantes mencionan la importancia de la actividad deportiva y explican que se trata de una de las actividades que más interés personal genera dentro de la semana: Carola y Lucía practican natación en compañía de varias amigas dos veces por semana, mientras que Alicia y Bruno juegan juntos al pádel un día de la semana. Además, Alicia y Bruno realizan otras actividades deportivas 
durante el fin de semana de manera habitual tanto con el núcleo de la familia como con familiares cercanos en el caso de Bruno.

Así, Bruno participa en un club de esquí los fines de semana, al que asiste con su prima y varios amigos de la escuela y en el que tienen libertad para esquiar de manera autónoma durante unas horas. El mismo lo relata así:

Entonces el sábado a las 6:15 me levanto, me arreglo y tal y a las $7 \mathrm{~h} 15 \mathrm{~min}$. tenemos que estar, que nos recogen en [... ¿ ¿Sabes dónde está el Cañas y Tapas?, pues nos recoge ahí el bus y ahí como a las nueve y media o diez llegamos a Valdesqui, esquiamos solos de $10 \mathrm{~h}$ a $12 \mathrm{~h} 30 \mathrm{~min}$., y a las $12 \mathrm{~h} 30 \mathrm{~min}$. vamos a la cafetería, comemos media horita y tal [...] (Bruno).

De esta manera, la práctica deportiva emerge como una actividad que aunque implique asumir compromisos e insertarse en un nuevo entramado de horarios y programas pre-estructurados, es también un espacio que los casos estudiados han elegido y organizado de manera autónoma. Tanto Bruno como Carola comentan que fueron ellos los que eligieron el tipo de deporte que querían realizar y ellos mismo organizan estas actividades de ocio; asumen la responsabilidad de traslado en la medida de lo posible y se desplazan para asistir con otros iguales a las actividades deseadas.

\section{Otros tiempos y espacios de ocio}

El "resto" del tiempo libre del que disponen los participantes se estructura en principalmente en torno al hogar. Aunque tienen cierta libertad para moverse por sus respectivos barrios y realizan todo tipo de gestiones y recados sin acompańamiento adulto, la configuración del hogar y el equipamiento del que disponen facilitan que este espacio sea el elegido para otros momentos de ocio. Antes que salir a la calle a jugar o pasear, los cuatro participantes estudiados prefieren utilizar la red como ventana al mundo exterior y como herramienta de conexión con amigos o familiares; incluso con aquellos que se encuentran físicamente más cercanos.

Igualmente, en las entrevistas se mencionan diferentes actividades que se realizan durante el tiempo libre en días lectivos, como leer -actividad que realizan todos de manera regular, aunque Carola y Bruno muestran especial preferencia por ésta. En particular, la lectura ocupa un lugar complejo en sus vidas en tanto que surge como obligación, dado que sus respectivos centros educativos exigen un número de lecturas por curso (en 2 idiomas en el caso de Bruno y Alicia), pero, 
por otra parte, es recibida con agrado y asumida voluntariamente por varios de los chicos y chicas:

[...] pero a mí me gusta leer y me divierte pero cuando son libros que de verdad me gustan, y este libro por ejemplo (refiriéndose a la fotografía) me ha gustado mucho, me ha parecido muy... hombre divertido no porque trata sobre una bomba nuclear pero... muy interesante como te cuentan que van sobreviviendo y todo... y ... no sé me ha gustado mucho porque te va contando lo que él va sintiendo y todo ... no sé, me parece un libro muy interesante (Bruno).

Volviendo al uso de las tecnologías, todos hacen un uso generalizado de las redes sociales y acceden a internet para actividades sin relación con los deberes o lo académico. Sin embargo, en este caso vemos algo más de variabilidad en cómo gestionan estos usos y la supervisión que ejercen sus padres. Carola y Lucía acceden a Internet de manera relativamente supervisada si se trata de uso lúdico, pues su ordenador está situado cerca del de su madre, lo que hace que ella esté pendiente del uso que hacen del ordenador:

Lucia: Siempre tenemos que preguntar si podemos subir al ordenador y ella dice: "ipara qué?" por ejemplo al Tuenti, al MSN... no nos deja los días que tenemos clase, aunque algunas veces $\mathrm{mi}$ hermana sube para bajarse música y a lo mejor si una amiga le ha contestado en Tuenti mi madre le deja verlo...

E: ¿Entras mucho? (En Tuenti)

Carola: No, no me da tiempo, durante la semana no puedo. Pero algún día que puedo pues entro. Por ejemplo, un día no nos mandaron $[\ldots]$ deberes y no tenía nada que hacer y $\mathrm{mi}$ madre me dejó ¡qué nunca me deja!

E: ¿уy eso?

C: Porque dice que tengo que estudiar...

E: ¿con quién estás cuando te metes?

C: a ver... es que mi ordenador está cerca del de mi madre y siempre estamos las dos...

Por el contrario, Alicia y Bruno lo hacen indistintamente y sus padres no están presentes cuando hacen uso de Internet. Alicia comenta y fotografía de la siguiente manera la escena: 


\section{Figura 4}

Uso de las TIC's sin supervisión

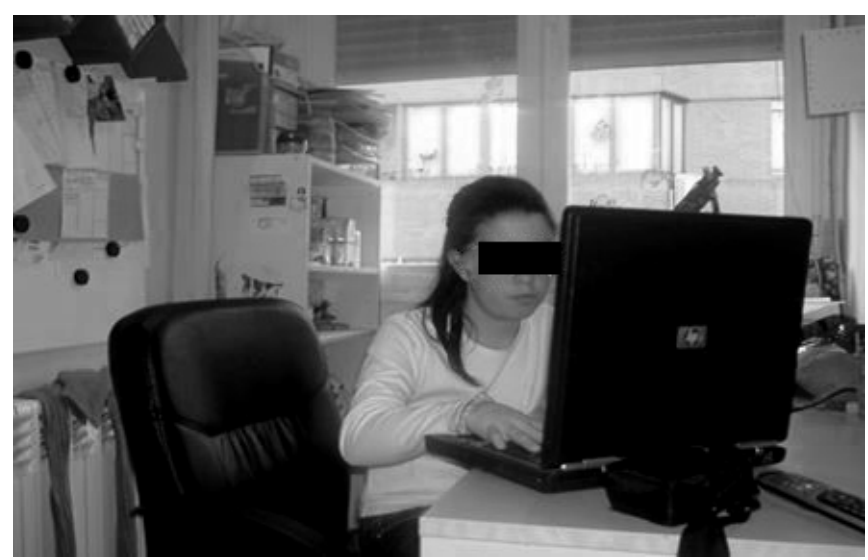

[...] un día después de hacer los deberes, yo creo, que me puse a buscar una cosa en el ordenador [...] a veces en los deberes nos ponen cosas que, que tenemos que buscar en el ordenador porque en el libro no salen [...] mientras te encuentra y tal, pues te metes en el Msn, miras a ver lo que pasa en Tuenti, es decir que normalmente no se puede separar con claridad el tiempo dedicado a trabajar y a cosas más personales. (Alicia).

Finalmente, todos los casos examinados mencionan la música como una parte relevante de su tiempo libre. Además, este consumo de música puede ser compartido y parte de la actividad familiar o puede ser individual. Carola escucha música con su hermana y su madre, cuando han terminado las tareas del día y están las 3 juntas preparando la cena. Forma parte del ritual diario en el que se junta la familia.

\section{El tiempo en familia}

En toda la discusión anterior la familia está siempre presente de forma más o menos explícita, en tanto que son co-participantes de las actividades, forman parte del espacio doméstico o incluso de otros espacios organizativos externos al hogar (e. g. el "club"). Los cuatro casos tematizan de manera explícita el tiempo en familia e identifican momentos en los que la actividad se estructura como "familiar" y esta emerge como la unidad social en situaciones en las que esperaríamos (especialmente en grandes entornos urbanos/metropolitanos) que 
aparecieran amistades sin vínculos de parentesco: la escuela, las actividades del fin de semana o la estructuración del tiempo libre están cruzadas por relaciones familiares. Además, entre los casos examinados encontramos diferencias en cuanto al papel que ocupa la familia extensa o la familia nuclear.

Alicia y Bruno tomaron fotografías del tiempo que pasan en casa de los abuelos y lo describen de manera similar. Igualmente, destacan las relaciones entre primos de edades similares como una parte muy importante de su red de iguales:

\begin{abstract}
Entonces, cada vez que hay un cumpleańos, aunque sea en invierno, como esta vez o lo que sea, mi abuela siempre hace una tarta y entonces todo, se soplan las velas todos juntos y todo es muy tradicional. Y luego [...] los que somos más pequeños de 17 comemos pizza o sea hay pizza ahí y tú te coges tu trozo, lo que quieras $[. .$.$] a mi me gusta mucho estar allí porque se reúnen$ todos los primos entonces como durante la semana hay algunos que no van a nuestro mismo colegio [...] (Bruno).
\end{abstract}

Por su parte, Alicia menciona a una prima de su edad con la que tiene una relación especial: ella y su prima juegan a colegios y preparan actividades para sus hermanas menores, que son "ejercicios que sé que ellas van a entender y digo, pues lo hacen así". Además, otros primos o tíos aparecen en las rutinas en días lectivos como Bruno y su madre los días que ambos juegan al pádel. En la narración sobre la vida familiar de Carola y Lucía pone mayor énfasis en la familia nuclear. La mayor parte de las actividades ocurren dentro del hogar y aunque es verdad que los primos aparecen como compañeros de juego durante el fin de semana, su presencia no es tan clara y el papel que otorgan a la familia extensa en su vida diaria queda algo más desdibujado - y aquí debemos destacar que el contraste es en cómo es narrada esta relación, mientras que "objetivamte" quizás los cuatro casos tengan un contacto con la familia extensa similar (e. g. ver a sus primos prácticamente todos los fines de semana) para cada chico o chica, este entramado familiar tiene un sentido diferente.

Las referencias a la familia a lo largo de las entrevistas de Carola y Lucía hacen alusión a las hermanas o a la madre en las rutinas diarias e incluso las relaciones de amistad cruzadas entre madre y hermanas, como habla Lucia: "Normalmente no vemos mucho la tele, porque cuando terminamos de cenar y hemos recogido nos metemos en la cama con mi madre y nos ponemos a hablar y a cotillear".

En resumen, el análisis conjunto de cómo los cuatro participantes de este trabajo organizan su actividad diaria en torno a las responsabilidades del hogar, el centro educativo, su ocio y vida familiar, da una visión del modo en que se configura la relación ciudad-hogar-actividad en niños y pre-adolescentes del entorno 
socio-cultural aquí presentado (familias madrileñas de clase alta residentes en los "barrios nobles" del centro urbano). En la discusión final retomamos algunos de los elementos que señalamos en la introducción y específicamente tratamos cómo esta organización material-espacial de la vida diaria contribuye a la reproducción del capital social y "estatus socio-cultural" de los participantes.

\section{Discusión y conclusiones}

Del análisis de la información que los participantes nos proporcionaron, podemos especificar dos ejes fundamentales que estructuran las actividades de los niños.

\section{La familia como espacio organizador de las actividades}

La familia constituye un eje fundamental dentro de la organización de las rutinas. Todos participantes se pudo observar que la familia juega un papel relevante en la organización de las actividades que ellos realizan. Pero además elaboran un discurso similar al explicar aquellos aspectos en los que está implicada la familia: consideran que forman parte de un modelo tradicional de familia en el que los abuelos, los primos y tíos juegan un papel relevante dentro de sus rutinas y en el que las fronteras entre los diferentes núcleos familiares se traspasan sin problemas.

Aunque se dan diferencias en la percepción de la familia entre Alicia y Bruno y, Carola y Lucía, desde su discurso se puede entender que la familia, sus prácticas y sus familias juega un papel fundamental dentro de sus vidas y que, aunque su presencia varíe, los miembros familiares están unidos por lazos muy estrechos y nexos de amistad, y de otras actividades, además de los propiamente familiares.

\section{La relevancia del hogar como proveedor de recursos}

En segundo lugar, el análisis nos muestra el hogar como la fuerza centrípeta desde la cual se organizan las actividades semanales. A los participantes se les proporcionó una cámara para fotografiar su rutina y, teniendo la libertad para realizar fotografías en cualquier lugar, el hogar se descubre como principal fuente de documentación. 
Aunque desde su discurso podemos entender que sus rutinas se dividen en diferentes espacios, el hogar es el núcleo desde el cuál se dan todas las demás actividades. Autores como Poveda et al. (2007) o Rasmussen (2004), descubren diferentes espacios de movilidad para la infancia. Sin embargo, los participantes de nuestro estudio organizan sus actividades entorno al hogar. Los participantes realizan actividades tanto en la escuela, como en instituciones reguladas, como en la calle, como en el hogar, pero todas ellas se organizan desde la lógica de los recursos que se obtienen dentro de este último.

El hogar se destapa como un espacio privilegiado y multiequipado en el que se pueden desplegar todos los gustos y que ofrece la protección necesaria para realizar aquellas actividades preferidas en cada caso concreto: la lectura, ver la televisión, uso de nuevas tecnologías, mantener contactos sociales. Desaparece así la necesidad de acceder a determinadas actividades a través de la oferta pública puesto que cualquier deseo o necesidad se puede cubrir desde todas las posibilidades que se aprecian en los hogares. Además, los participantes encuentran en el hogar las ventanas necesarias como Internet, teléfono, etc. para estar en contacto con el espacio público pero desde la comodidad de lo privado.

En estos casos, en contraposición a lo que tradicionalmente se creía, no es únicamente la configuración de las ciudades la que lleva a la infancia al espacio privado, si no que éstos se eligen de manera personal, dadas las posibilidades que ofrece como puente hacia otras esferas, como contacto con los iguales o como lugar en el que desarrollar actividades de tiempo libre.

Cabe destacar que en los 3 casos familiares estamos hablando de situaciones en las que el capital económico proporciona un grado amplio de formación a nivel familiar. No sólo los participantes tienen acceso a todo tipo de recursos, si no que sus padres, y su entorno próximo, disfruta de un alto capital económico y cultural que asimismo facilita la formación de los hijos y les permite acceder a una amplia gama de recursos dentro del hogar.

Siguiendo a Bourdieu (1994, p. 135), una de las características típicas:

[...] de los dominantes consiste en tener familias particularmente extensas (los grandes tienen familias grandes) y fuertemente cohesionadas, en tanto que unidas no sólo por la afinidad de los hábitos sino también por la solidaridad de los intereses [...] el capital económico evidentemente pero también el capital simbólico, y sobre todo, tal vez, el capital social.

En este sentido el capital económico y cultural de la familia permiten el desarrollo y el acceso a determinados recursos que generalmente se limitan a la elite cultural. La familia se constituye como la primera fuente de transmisión y consumo cultural y como instrumento de construcción de la realidad. (BOURDIEU, 1994) 
Teniendo en cuenta que la familia es el principal agente de socialización y que el hogar es el espacio en el que se producen las relaciones familiares, ambos aspectos posibilitan la autonomía y gestión de los recursos que hacen los participantes.

En línea con Bourdieu Gubbins (2011) y Lareau (2002) muestran la importancia de las condiciones económicas y socio históricas del grupo estudiado y cómo el hogar y la familia surgen como apoyo privilegiado que fomenta la autonomía y la formación de los menores dentro de un ambiente de protección en el que desplegar sus actividades con todas las facilidades y recursos posibles.

Y los participantes así lo valoran y lo reconocen. En los casos estudiados el hogar les ofrece la posibilidad de desplegar su propia identidad, dentro de lo que ellos mismos reconocen como una aspecto de su identidad familiar, en espacios diferenciados y específicamente acondicionados para ello: el cuarto de juego, la sala de estar, su propia mesa de estudio etc.

Por tanto, retomando los aspectos señalados en la introducción, vemos que si bien la bibliografía tradicional sobre la infancia en la ciudad se incide en que la infancia ha sido relegado al hogar por una ciudad que no les proporcionaba zonas específicamente diseńadas para ellos, en nuestro caso, nuestros participantes no percibían la ciudad como un espacio hostil o no apto para ellos, si no que su entorno se consideraba favorable. Al contrario de lo que documenta otros estudios de la ciudad de Madrid, los nińos no perciben su entorno como un lugar peligroso en el que deben pelear por los recursos, más bien se mueven sin dificultades por el distrito y muestran seguridad sobre sus desplazamientos.

Pero, sin embargo a través de las fotografías y entrevistas realizadas, identificamos que ocio y tiempo libre no se estructura únicamente entorno al espacio público. Hogar y familia juegan un papel relevante en las actividades de las niñas y se deben considerar como dos de los ejes principales sobre los que se construyen las actividades diarias.

En primer lugar, y como hemos comentado, el acceso de la infancia a ciertos recursos permite que el hogar se convierta en eje que estructura sus rutinas y en la ventana idónea para organizar otras actividades gracias a Internet y demás medios digitales como teléfonos, ordenadores portátiles, videojuegos, etc., que encuentran dentro del espacio privado, sin necesidad de realizar una búsqueda en el espacio público. Algo que desdibuja la dicotomía espacio público-espacio privado y que es configurado por las niñas a través de sus usos y prácticas por encima de los espacios físicos. (POVEDA et al, 2012; GONZÁLEZ-PATIÑO, 2011)

Sin embargo, en nuestra comprensión de las rutinas infantiles cobra relevancia su acceso al capital cultural y la gestión que realizan del mismo. Según la lógica de Bourdieu que comentan otros autores como Lenoir (2005) las personas acceden a determinadas élites no tanto por aquellos recursos que se adquieren en la escuela, o en instituciones públicas, si no que el capital se hereda dentro de la 
familia y se puede entender desde su trayectoria social y la de los demás miembros de la familia, incluyendo aquí abuelos, tíos, parientes próximos etc.

Quizás, en este sentido sería interesante documentar las rutinas entre niños pertenecientes a otras clases sociales y distritos con distintos accesos a recursos y equipamientos del hogar diferenciados, ya que nos permitiría comprobar su movilidad. Debemos tener en cuenta, que no sólo la ciudad modula los hogares, si no que la relación se produce también a la inversa, es decir, los hogares modulan nuestra percepción de la ciudad. Por lo tanto, acercarnos a otro tipo de hogares nos permitiría documentar otras maneras de explorar la ciudad. Ver como niños de diferentes estratos socio-económicos asumen o no las estrategias familiares en la reproducción de las metas de progreso y/o conservación del orden socioeconómico. Las niñas tienen acceso a redes sociales, chats, información online, etc. lo que les permite estar permanentemente conectados al exterior sin la necesidad de "salir a la calle" de la manera más tradicional explorando nuevas posibilidades, a las tradicionales formas de relación en la ciudad. Los niños no se ven como personas relegadas al ámbito privado, si no que éste ha sido elegido con total libertad, puesto que les permite realizar todas aquellas actividades que desean para desarrollar sus gustos y gestionar su tiempo libre de manera autónoma, continuando en momentos con las prácticas familiares y en ocasiones llevándolas más allá.

Por ello, es interesante estudiar las nuevas posibilidades que ofrece los diferentes medios digitales para establecer contactos, adquirir conocimientos... Pero también, como hemos explorado en otros trabajos, aquellos momentos de resistencia o identificación a los valores familiares (GONZÁLEZ- PATIÑO, 2011; ALONSO, 2010), más allá de las estrategias socioeducativas familiares y/o escolares.

\section{Referências}

ALONSO, B. Análisis de la identidad a través del discurso: Auto-retrato de un preadolescente y posicionamientos familiares. 2010. Facultad de Psicología, Universidad Autónoma de Madrid. [Tesina no publicada].

BOURDIEU, P. El espíritu de la familia. In: Razones prácticas: sobre la teoría de la acción. Barcelona: Ediciones Anagrama, 1994, p. 126-138.

CAPPELLO, M. Photo interviews: eliciting data through conversations with children. Field Methods, London, v. 17, n. 2, p. 170-182, may. 2005.

COLEMAN, J. et al. Equality of Educational Opportunity. Washington, D.C.: U.S. Dept. of Health, Education, and Welfare, Office of Education, 1966. 
COOK, T.; HESS, E. What the camera sees and from whose perspectives. Fun methodologies for engaging children in enlightening adults. Childhood, London and New Delhi, v. 14, n. 1, p. 29-45, feb. 2007.

CHRISTENSEN, P; JAMES, A.; JENKS, C. Home and Movement: children constructing 'family time'. In: HOLLOWAY, S.; VALENTINE, G. (Eds). Children's Geographies: playing, living and learning. London: Routledge, 2000. p. 139-155.

DE CARVALHO, M. P. Rethinking family-school relationships: a critique of parental Education. New York: Free Press, 2001.

GONZÁLEZ-PATIÑO, J. Rutinas de la infancia urbana mediadas por la tecnología: un análisis visual. Papeles de Trabajo sobre Cultura, Educación y Desarrollo Humano, Madrid, v. 7, n. 2, p. 1-16, jul. 2011.

GUBBINS, V. Estrategias de Involucramiento Parental en familias de estudiantes con buen rendimiento escolar en educación básica. 2011. Tesis [Doctora en Ciencias de la Educación]. Pontificia Universidad Católica de Chile, Santiago. 2011.

LAREAU, A. Invisible Inequality: Social Class and Childrearing in Black Families and White Families. American Sociological Review, Washington DC, v. 67, n. 5, p. 747-776, oct. 2002.

LENOIR, R. La genealogía de la moral familiar. Political y Sociedad, Madrid, v. 42, n. 3, p. 209-225, 2005. [Centre de Sociologie Européene (CNRS- EHESS); Universidad de París I (Pantheón- Sorbonne)].

POVEDA, D. et al. The after school routines of literature-devoted urban children. Children's Geographies, London, v. 5, n. 4, 423-441, dec. 2007.

POVEDA, D; MORGADE, M.; GONZÁLEZ-PATIÑO, J. Children at home in Madrid. ETNIA-E: Cuadernos de Investigación Etnográfica sobre Infancia, Adolescencia y Educación del IMA/FMEE, n. 4, p. 1-15, 2012. Disponible en http://e-revistas.ima.org.es. Acceso en 29 dec. 2013.

RASMUSSEN, K. Places for children-children's places. Childhood, Norway, v. 11, n. 2, p. 155-173, May. 2004.

ROGOFF, B. The cultural nature of human development. Oxford: Oxford University Press, 2003.

VINCENT, C. et al. Being Strategic, Being Watchful, Being Determined: black middle class parents and schooling. British Journal of Sociology of Education, London, v. 33, n. 3, p. 337-354, apr. 2012.

Recebido em 17 de julho de 2014.

Aprovado em 24 de outubro de 2014. 